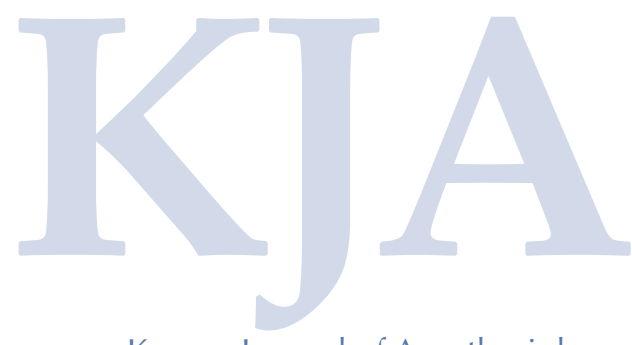

Korean Journal of Anesthesiology

\title{
Spinal anesthesia for cesarean
} section in a patient with systemic sclerosis associated interstitial lung disease

\section{-a case report-}

\author{
Guie Yong Lee and Sooyoung Cho \\ Department of Anesthesiology and Pain Medicine, Ewha Womans University School of Medicine, Seoul, Korea
}

Systemic sclerosis or scleroderma is a rare autoimmune disorder characterized by excessive fibrosis and, vasculopathy, with multiorgan involvement. Anesthetic considerations in patients with systemic sclerosis must take into account the degree of organ dysfunction as well as airway management. Regional anesthesia is a preferable alternative to general anesthesia despite the reports of prolonged sensory block. Spinal anesthesia in patients with systemic sclerosis has been reported for only one patients undergoing cesarean section. Concurrent systemic sclerosis and pregnancy raise many obstetric and anesthetic considerations. We describe the case of a pregnant patient with systemic sclerosis who had a history of dyspnea and interstitial lung disease. The cesarean section was performed uneventfully under spinal anesthesia.

Key Words: Cesarean section, Interstitial lung disease, Spinal anesthesia, Systemic scleroderma.

Systemic sclerosis (SSc) or scleroderma is a multisystem autoimmune disease characterized by excessive collagen deposition in the skin and internal organs, and microvascular changes. Anesthesiologists face difficulties with anesthetic management during surgical procedures for patients with SSc due to problems involving the lungs, heart, gastrointestinal tract, and kidneys as well as difficult airway management. SSc is three times more

Corresponding author: Guie Yong Lee, M.D., Ph.D.

Department of Anesthesiology and Pain Medicine, Ewha Womans University School of Medicine, 1071, Anyangcheon-ro, Yangcheongu, Seoul 07985, Korea

Tel: 82-2-2650-5285, Fax: 82-2-2655-2924

E-mail: lgyanes@ewha.ac.kr

Received: July 20, 2015.

Revised: September 2, 2015.

Accepted: September 7, 2015.

Korean J Anesthesiol 2016 August 69(4): 406-408 http://dx.doi.org/10.4097/kjae.2016.69.4.406 common in women than in men [1] and 15 times more frequent in women in the childbearing years [2]. Adverse obstetric outcomes in SSc include miscarriages, preterm births, intrauterine growth restriction, and cesarean deliveries [3,4]. Because pregnant patients with SSc are uncommon, published reports of anesthetic management for cesarean sections and epidural analgesia for labor have been limited. There have been no case reports focusing on spinal anesthesia for cesarean sections since the description of Bailey et al. [5] was published in 1999. Here, we describe the case of a pregnant patient with SSc-associated interstitial lung disease (ILD) who underwent cesarean section under spinal anesthesia.

The patient permitted publication of a case report.

\section{Case Report}

A 40-year-old multiparous woman $(164 \mathrm{~cm}, 74 \mathrm{~kg})$ at 38 weeks gestation was admitted for a repeat cesarean section. She had been diagnosed with SSc twelve years earlier. The patient

(c) This is an open-access article distributed under the terms of the Creative Commons Attribution Non-Commercial License (http://creativecommons.org/ licenses/by-nc/4.0/), which permits unrestricted non-commercial use, distribution, and reproduction in any medium, provided the original work is properly cited. 
had no known hereditary disease. She had experienced dyspnea on exertion for 10 years, and ILD had been diagnosed with a lung biopsy one year previously. A preoperative chest radiograph revealed bilateral basal pulmonary fibrosis. The pulse oximetry $\left(\mathrm{SpO}_{2}\right)$ was $95 \%$ with the patient breathing room air in the antenatal care unit, and pulmonary function tests showed decreased forced vital capacity (FVC)\% predicted (60\%), diffusing capacity of the lungs for carbon monoxide (DLCO)\% (64\%), and forced expiratory volume in 1 second (FEV1) (57\%). Arterial blood gas analysis results were as follows: fraction of inspired oxygen 0.21 , pH 7.44, $\mathrm{PaO}_{2} 89.9$ mmHg, $\mathrm{PaCO}_{2} 32.2 \mathrm{mmHg}, \mathrm{HCO}_{3}{ }^{-} 21.6$ $\mathrm{mmol} / \mathrm{L}, \mathrm{SaO}_{2} 96.7 \%$. The patient was receiving prednisolone 5 $\mathrm{mg}$ /day due to migrating joint pain during pregnancy, and she was taking an antacid for gastroesophageal reflux. The coagulation test results were normal. On preoperative examination, the patient had no limitation of mouth opening and was given a Mallampati score of class 2. Following admission, the patient was administered oxygen 3 liters per minute via nasal catheter. Consultation from the pulmonology department estimated that the risk of postoperative pulmonary complications is five times higher in patients with ILD compared to the general population, associated with the possibility of acute deterioration of interstitial fibrosis. After discussion with the patient, the obstetric surgeon and the anesthesiologist, the decision was made to perform the procedure with combined spinal epidural (CSE) anesthesia. The patient received $100 \mathrm{mg}$ of hydrocortisone before arrival in the operating room.

Upon arrival in the operating room, the noninvasive blood pressure (NBP) was $107 / 56 \mathrm{mmHg}$ and the heart rate was 60 beats/min. Five hundred $\mathrm{ml}$ of normal saline solution was infused before the spinal injection. Standard monitoring was used throughout the operation, including electrocardiography, NBP monitoring, and pulse oximetry. With the patient in the right lateral recumbent position, an $18 \mathrm{G}$ Tuohy and $27 \mathrm{G}$ pencil point spinal needle were inserted via the needle-through-needle technique at the L3-4 interspace. A free-flow of cerebrospinal fluid was observed before $1.3 \mathrm{ml}$ heavy bupivacaine $0.5 \%$ was injected. There was a failure of advancement of the epidural catheter with two attempts at another interspace. Fifteen minutes after the spinal injection of heavy bupivacaine, the sensory block (pinprick test) obtained to T5 with complete motor block of the lower extremities. The blood pressure decreased to $72 / 48 \mathrm{mmHg}$ 25 minutes after the spinal injection and remained stable after intravenous ephedrine $10 \mathrm{mg}$ and the infusion of additional fluids. A male baby $(2.63 \mathrm{~kg}$ ) was born with Apgar scores of 9 and 10 at 1 and 5 minutes respectively. The sensory level was regressed to T8 and T10 after 90 minutes (at the end of the operation) and 140 minutes (discharge from the postanesthesia care unit), respectively. The motor block level regressed to Bromage 0 (left leg) and 1 (right leg) at discharge from the postanesthesia care unit. When visited on postoperative day 1 , the patient was in good condition. She was discharged on postoperative day 4.

\section{Discussion}

Pregnant women with SSc are considered high risk patients, and they pose a potential anesthetic challenge during labor analgesia and operative delivery. If a cesarean section is planned, a series of preoperative laboratory tests, including a complete blood cell count, urinalysis and creatinine levels, electrolytes, albumin levels, coagulation screen, electrocardiogram, chest radiograph, arterial blood gas analysis, and pulmonary function test, should be performed [6]. Echocardiography is now more frequently employed as it is useful in the assessment of ventricular dysfunction, pericardial and pleural effusions, and pulmonary hypertension [7].

Our patient had dyspnea for 10 years, and was diagnosed with ILD via a lung biopsy. A preoperative chest radiograph revealed bilateral basal pulmonary fibrosis. Pulmonary function tests showed a moderate pulmonary insufficiency of mixed type and mildly decreased diffusing capacity of the lungs for carbon monoxide. The $\mathrm{SpO}_{2}$ measured in the antenatal care unit showed $95 \%$. ILD is common and a leading cause of morbidity and mortality in patients with SSc. Patients with mild interstitial involvement are asymptomatic during the early stages, and clinically significant ILD is observed in approximately $40 \%$ of patients with SSc $[8,9]$. Pulmonary function tests are a principal component in the diagnosis and prognosis of SSc-ILD. Pulmonary function tests shows restrictive changes with reduced FVC and DLCO in SSc $[9,10]$. Arterial hypoxemia resulting from reduced diffusing capacity is common in these patients [11]. If a patient's vital capacity is lower than $1 \mathrm{~L}$, weaning and extubation are difficult due to an inadequate ventilator effort during the postoperative period following general anesthesia. Moreover, such patients require increased positive airway pressure and a higher than normal oxygen concentration for adequate ventilation and diffusion [12].

There are no specific choices for the type of anesthesia in patients with SSc. If general anesthesia is required, the patient should be prepared for difficult intubation and aspiration of gastric contents. Furthermore, a thorough preoperative physical examination should be performed to screen for skin tightening in the face and neck and limitation of mouth opening, as these can affect intubation. Selection of anesthetic agents should be determined on an individualized basis, according to the level of organ involvement.

Regional anesthesia is a preferable alternative to general anesthesia and is also useful for postoperative analgesia. Moreover, the vasodilatory effects of sympathetic blockade can improve tissue perfusion and prevent Raynaud's phenomenon. However, 
several case studies have reported problems of prolonged sensory block as a result of abnormal responses to local anesthetics in patients with SSc. Since 1964, patients in seven out of 21 cases of local and regional anesthesia in individuals with SSc have shown a prolonged sensory block. Six of these seven patients had prolonged peripheral nerve block and only one had a labor epidural block using chloroprocaine [13]. Thompson and Conklin [14] reported that T6-S5 anesthesia was maintained for 5 hours and 55 minutes after the injection of $320 \mathrm{mg}$ of chloroprocaine. However, a prolonged block has not been reported since 1999, when spinal, epidural, CSE anesthesia, and continuous intrathecal catheter techniques were performed using bupivacaine, ropivacaine, and prilocaine [13]. Spinal anesthesia was performed in only one parturient patient undergoing a cesarean section. Bailey et al. [5] reported that when a patient with pre-eclampsia with limited mouth opening and a class 4 Mallampati score underwent subarachnoid injection of $4.75 \mathrm{ml}$ of $0.25 \%$ plain bupivacaine with $0.25 \mathrm{mg}$ diamorphine, the sensory block reached the T2 level and full sensation was recovered 3.5 hours later. However, the patient experienced severe hypotension $(160 / 90 \mathrm{mmHg}$ to systolic pressure $90 \mathrm{mmHg}$ ) shortly after establishment of the block and was administered a total of $42 \mathrm{mg}$ of ephedrine. The authors suggested that the precipitate hypotension was induced by chronic vasoconstriction and relative hypovolemia due to SSc and pre-eclampsia. In the present case, the CSE technique was chosen in consideration of a risk of postoperative pulmonary complications and a possibility of acute deterioration of interstitial fibrosis. However, only a single shot spinal anesthesia was used to maintain the block after the failure to insert the catheter into the epidural space. A mixture of fentanyl and ketorolac was intravenously infused for postoperative analgesia. The patient in the present case showed similar patterns of sensory and motor block recovery to those of normal parturients until discharge from the postanesthesia care unit, and she was able to perform activities such as breast-feeding without any particular differences compared to normal parturients. A limitation of this case study may be the fact that we did not record the accurate times of full sensory and motor block recovery after the patient was discharged from the postanesthesia care unit.

In conclusion, our patient demonstrates an example of uneventful management of spinal anesthesia during a cesarean section. However, due to the rarity of reports of anesthesia in pregnant women with SSc, we recommend that anesthesiologists should keep in mind the possibility of abnormal responses to local anesthetics.

\section{References}

1. Chifflot H, Fautrel B, Sordet C, Chatelus E, Sibilia J. Incidence and prevalence of systemic sclerosis: a systematic literature review. Semin Arthritis Rheum 2008; 37: 223-35.

2. Steen VD, Medsger TA Jr. Epidemiology and natural history of systemic sclerosis. Rheum Dis Clin North Am 1990; 16: 1-10.

3. Steen VD. Pregnancy in scleroderma. Rheum Dis Clin North Am 2007; 33: 345-58, vii.

4. Chakravarty EF, Khanna D, Chung L. Pregnancy outcomes in systemic sclerosis, primary pulmonary hypertension, and sickle cell disease. Obstet Gynecol 2008; 111: 927-34.

5. Bailey AR, Wolmarans M, Rhodes S. Spinal anaesthesia for caesarean section in a patient with systemic sclerosis. Anaesthesia 1999; 54: 355-8.

6. Pophan P, Reid RW. Autoimmune disorders. In: Chestnut's Obstetric Anesthesia: Principles and Practice. 5th ed. Philadephia, Elsevier Saunders. 2014, pp 947-59.

7. Plastiras SC, Papazefkos V, Pamboucas C, Sfikakis P, Toumanidis S. Scleroderma heart: pericardial effusion with echocardiographic signs of tamponade during pregnancy. Clin Exp Rheumatol 2010; 28: 447-8.

8. Highland KB, Silver RM. New developments in scleroderma interstitial lung disease. Curr Opin Rheumatol 2005; 17: 737-45.

9. Schoenfeld SR, Castelino FV. Interstitial lung disease in scleroderma. Rheum Dis Clin North Am 2015; 41: 237-48.

10. Herzog EL, Mathur A, Tager AM, Feghali-Bostwick C, Schneider F, Varga J. Review: interstitial lung disease associated with systemic sclerosis and idiopathic pulmonary fibrosis: how similar and distinct? Arthritis Rheumatol 2014; 66: 1967-78.

11. Sackner MA, Akgun N, Kimbel P, Lewis DH. The pathophysiology of scleroderma involving the heart and respiratory system. Ann Intern Med 1964; 60: 611-30.

12. Roberts JG, Sabar R, Gianoli JA, Kaye AD. Progressive systemic sclerosis: clinical manifestations and anesthetic considerations. J Clin Anesth 2002; 14: 474-7.

13. Dempsey ZS, Rowell S, McRobert R. The role of regional and neuroaxial anesthesia in patients with systemic sclerosis. Local Reg Anesth 2011; 4: 47-56.

14. Thompson J, Conklin KA. Anesthetic management of a pregnant patient with scleroderma. Anesthesiology 1983; 59: 69-71. 